\title{
Pyruvate is a prospective alkalizer to correct hypoxic lactic acidosis
}

\author{
Ying Wang ${ }^{1}$, Ya Huang ${ }^{1,2}$, Jing Yang ${ }^{1}$, Fang-Qiang Zhou ${ }^{3,4}$, Lian Zhao ${ }^{1 *}$ and Hong Zhou ${ }^{1 *}$
}

\begin{abstract}
Type A lactic acidosis resulted from hypoxic mitochondrial dysfunction is an independent predictor of mortality for critically ill patients. However, current therapeutic agents are still in shortage and can even be harmful. This paper reviewed data regarding lactic acidosis treatment and recommended that pyruvate might be a potential alkalizer to correct type A lactic acidosis in future clinical practice. Pyruvate is a key energy metabolic substrate and a pyruvate dehydrogenase (PDH) activator with several unique beneficial biological properties, including anti-oxidant and antiinflammatory effects and the ability to activate the hypoxia-inducible factor-1 (HIF-1a) - erythropoietin (EPO) signal pathway. Pyruvate preserves glucose metabolism and cellular energetics better than bicarbonate, lactate, acetate and malate in the efficient correction of hypoxic lactic acidosis and shows few side effects. Therefore, application of pyruvate may be promising and safe as a novel therapeutic strategy in hypoxic lactic acidosis correction accompanied with multi-organ protection in critical care patients.
\end{abstract}

Keywords: Type A lactic acidosis, Hyperlactatemia, Pyruvate, Glucose metabolism, PDH activator

\section{Background}

Lactic acidosis commonly occurs in numerous clinical conditions with severe pathogenic insults and indicates a serious prognosis. Lactic acidosis is referred to as acidosis with hyperlactatemia in terminology and is defined as metabolic acidosis with a serum lactate concentration $>4$ or $5 \mathrm{mmol} /$ $\mathrm{L}$ and an arterial $\mathrm{pH}(\mathrm{pHa})<7.35$. There are traditionally two categories of lactic acidosis: type A and type B. Type A lactic acidosis (LA) primarily results from hypoxic mitochondrial dysfunction, which causes lactate accumulation. It is a result associated with insufficient supply and underutilization of oxygen in tissues. In clinical settings, LA often appears as one of the severe complications in patients subjected to hypotension, as with hemorrhage, trauma and sepsis, and in patients with weakened oxygenation, such as with carbon monoxide, cyanide and iron poisoning, severe anemia, exhausted muscle activity (exercise, asthma) and hemoglobin-transfer disorders $[1,2]$. Type B has a characteristic of hyperlactatemia, but clinical evidence of tissue hypoxia is absent. Type B is subdivided into B1 (associated with certain underlying diseases such as liver failure), B2

\footnotetext{
* Correspondence: zhaolian@bmi.ac.cn; zhouhtt1966@163.com ${ }^{1}$ Institute of Transfusion Medicine, Academy of Military Medical Sciences, Beijing 100850, China

Full list of author information is available at the end of the article
}

(due to drugs or toxins) and B3 (caused by inborn errors of metabolism) [2]. In the present paper, we mainly discussed the treatment of LA specifically referring to type A.

\section{Lactic acidosis (type A) in clinical settings}

It has been long known that LA is an independent predictor of mortality for critically ill patients. A high lactate level is closely correlated with a poor prognosis in patients subjected to severe hemorrhage (HS). Abramson et al. [3] provided early evidence that trauma patients whose lactate levels were normalized (serum levels $<2 \mathrm{mmol} / \mathrm{L}$ ) within the first $24 \mathrm{~h}$ in the intensive care unit (ICU) were more likely to survive, those who normalized between 24 and $48 \mathrm{~h}$ had a $25 \%$ mortality and those who normalized over $48 \mathrm{~h}$ after injury had a nearly $86 \%$ mortality. Furthermore, initial and peak lactate levels closely correlate with multiple organ dysfunction syndrome and survival rate following trauma [4]. Notably, relative hyperlactatemia in the normal reference range is also independently associated with increased hospital mortality in critical care patients [5]. Currently, the incidence of hyperlactatemia is over $30 \%$ and the mortality rate of LA is over $50 \%$ in children and adults in ICUs. The European guideline on the management of major bleeding and coagulopathy following trauma (fourth edition) [6] recommends serum lactate 
and/or base deficit measurements as sensitive tests to estimate and monitor the extent of bleeding and shock (Grade 1B). Early lactate-guided therapy is a beneficial method established for reducing sepsis mortality [7] and improving the outcome of critically ill patients [8]. However, current therapeutic agents are still in shortage and even somewhat harmful for LA treatment and the effort to discover effective agents remains necessary. In this paper, we reviewed data regarding LA treatment in animal experiments and clinical settings and strongly recommended that pyruvate as an optimal candidate might be a prospective alkalizer to correct LA in future clinical practice.

\section{Pathophysiological alterations of lactic acidosis}

Type A LA is the direct result of cellular hypoxia. The main pathophysiological changes of LA are hypoxic metabolic disorders, which are mainly as follows.

\section{Inhibition of aerobic metabolism}

During aerobic metabolism (oxidative phosphorylation), lactate and hydrogen ions (proton $\left[\mathrm{H}^{+}\right]$) are consumed in the mitochondrial respiratory chain to produce $\mathrm{CO}_{2}$, $\mathrm{H}_{2} \mathrm{O}$ and adenosine triphosphate (ATP). However, oxidation in mitochondria is significantly inhibited during cellular hypoxia due to depressed activity of a key enzyme, pyruvate dehydrogenase (PDH) complex and an insufficient oxygen supply. Therefore, lactate cannot be oxidized and $\left[\mathrm{H}^{+}\right]$is accumulated. Both are transported out of cells into the tissue interstitial fluid via the monocarboxylic acid transporter $(\mathrm{MCT})$ and $\left[\mathrm{Na}^{+}\right] /\left[\mathrm{H}^{+}\right]$ exchanger, resulting in hyperlactatemia and acidosis.

\section{Promotion of glycolysis}

Hypoxia prompts anaerobic glucose metabolism, known as glycolysis, which produces two ATP molecules from one glucose molecule (2 glycolytic ATPs vs. 30 mitochondrial ATPs in aerobic metabolism). Endogenous pyruvate is converted by lactate dehydrogenase (LDH) into lactate, instead of into acetyl-CoA, in the glycolytic pathway. Prolonged and severe tissue hypoxia results in the generation of large quantities of lactate from anaerobic glycolysis and excessive $\left[\mathrm{H}^{+}\right]$from glycolytic ATP hydrolysis, or water dissociation according to Stewart theory. In addition, clinical use of adrenaline may also promote glycolysis to produce lactate.

\section{Reduction of lactate clearance/conversion}

Lactate is produced mainly from skeletal muscle, skin, brain, intestines and erythrocytes [9]. Under pathological conditions, in which oxygen delivery is limited, lactate generation increases. A decreased lactate clearance is therefore an additional cause of increased lactate concentrations. Lactate is metabolized primarily in the liver and kidneys. During critical conditions, hypoxia often induces organ damage and gluconeogenesis via the Cori cycle may be finally inhibited due to dysfunction of the liver and kidneys, leading to a further decrease of lactate clearance.

\section{Current non-ideal therapeutic agents Alkaline agents}

The administration of bases (alkalizers) is commonly regarded as the symptomatic treatment to correct metabolic acidosis, but it is unfavorable in LA corrections.

\section{Sodium bicarbonate (SB)}

Although it is not recommended in the majority of trauma guidelines, SB has been widely used to correct metabolic acidosis in clinical scenarios. Administration of SB can elevate the bicarbonate $\left(\left[\mathrm{HCO}_{3}{ }^{-}\right]\right)$level and raise the $\mathrm{pH}$ in plasma, which benefits patients with decreased serum $\left[\mathrm{HCO}_{3}{ }^{-}\right]$. However, SB may cause a series of complications. It increases carbon dioxide $\left(\mathrm{CO}_{2}\right)$ production and decreases serum ionized calcium, which contributes to a decrease in ventricular and vascular contractility and damage to the myocardium. More importantly, SB therapy may induce an intracellular $\mathrm{pH}(\mathrm{pHi})$ decline. It was reported that bicarbonate administration led to an average increase in $\mathrm{CO}_{2}$ generation reflected by the need to increase ventilation by $40 \%$ to maintain a stable $p \mathrm{CO}_{2}$ [10]. For patients with abnormal respiratory compensation, $\mathrm{CO}_{2}$ generated from SB can rapidly diffuse into cytoplasm, which causes overproduction of intracellular $\left[\mathrm{H}^{+}\right][10]$. The decreased pHi will worsen intracellular acidosis and damage tissues and organs. A randomized crossover study of 14 adults with lactic acidosis $\left(\left[\mathrm{HCO}_{3}{ }^{-}\right]<17\right.$ or base excess $<-10$ and lactate nearly $7.8 \mathrm{mmol} / \mathrm{L})$ treated with $\mathrm{SB}$ or equimolar sodium chloride demonstrated that SB treatment did not improve hemodynamics in ICU patients who suffered from LA [11]. It also cannot reduce but can even exacerbate the lactate accumulation [1]. Furthermore, SB has several negative side effects, such as altering blood pressure and triggering apoptosis [12]. SB administration requires a functional cardio-respiratory system to exhale the extra $\mathrm{CO}_{2}$ and there is a trend against using it in cardiac arrest patients. Therefore, SB use should be limited with caution to correct LA in critical care patients, i.e., avoiding the administration of $\mathrm{SB}$ if the $\mathrm{pHa}$ is $>7.10$.

\section{Tromethamine (THAM)}

THAM is an amino alcohol with acid-buffering capacity [1]. The $\mathrm{NH}_{2}$ in THAM can bind $\left[\mathrm{H}^{+}\right]$to modulate both $\mathrm{pHa}$ and $\mathrm{pHi}$ as well as blood $\mathrm{CO}_{2}$ levels. However, it does not affect lactate accumulation. THAM also has toxic side effects such as respiratory depression and hypoglycemia [13]; thus, it is not suitable for LA treatment [1]. Moreover, except in a few case reports, THAM is no longer available for clinical use in most parts of the world. 


\section{The activator of PDH}

$\mathrm{PDH}$ is a key enzyme that modulates glucose oxidation, which converts pyruvate to acetyl-CoA. During hypoxia, $\mathrm{PDH}$ activity is significantly inhibited and then pyruvate is converted into lactate. Therefore, PDH activators enable correction of LA because of their ability to accelerate pyruvate oxidation and improve metabolic disturbances.

Dichloroacetate (DCA), the representative activator of PDH, has been administered since at least 1978 to patients with inborn errors of mitochondrial metabolism and is able to lower lactate concentrations and normalize blood $\mathrm{pH}$. However, a large randomized controlled trial failed to demonstrate its effect on LA, illustrating that DCA increased arterial $\mathrm{pH}$ and decreased blood lactate but did not reduce mortality in ICU patients $[14,15]$. A further study showed that DCA reduced mitochondrial NADH and elevated the incidence of premature ventricular contractions when glucose was the only exogenous fuel in isolated rat hearts during normoxic perfusion, which was mitigated by the addition of PDH substrates such as pyruvate [16]. Therefore, when using DCA to correct LA, PDH substrates may be needed. In addition, DCA may be harmful because it causes neuropathy $[17,18]$. Nevertheless, the more rational treatment with appropriate doses of DCA to reduce side effects may need to be further investigated in patients with moderate or early-stage LA.

Other PDH activators, such as phenylbutyrate and desacylghrelin (DAG), are being studied [19, 20]. It was reported that phenylbutyrate increased the residual activity of PDH by increasing the proportion of unphosphorylated enzymes and had potential as a therapeutic agent for LA [21]. DAG, the precursor peptide of ghrelin, could normalize skeletal muscle lactate production and plasma lactate levels elevated by burn injury through the down-regulation of elevated inflammatory cytokines and activation of PDH [22]. However, the results were not ideal and further study is needed.

\section{Other chemicals}

Many other agents are being investigated to better manage LA. For example, the compound 5-amino-2-hydroxymethylphenyl boronic acid, a phenyl boronic acid derivative, binds lactate and normalizes the blood $\mathrm{pH}$ by increasing the consumption of protons via the LDH pathway [12]. Spermidine, with its activating effect on PDH phosphatase, can also activate PDH, stimulating the decarboxylation of pyruvate and inhibiting lactate accumulation [1]. In addition, NHE1 (a cell-membrane $\left[\mathrm{Na}^{+}\right] /\left[\mathrm{H}^{+}\right]$ exchanger) inhibitors may be useful in reverse of LA, but are still in animal studies [23]. Thus, these agents need more research to verify their effects and safety in patients.

\section{Anions in fluid therapy}

Resuscitation to support circulation is one of the first steps in treating LA [22]. Intravenous (IV) fluids were first administered over 180 years ago [23] and fluid infusion is considered the mainstay of therapy for critical care patients. The organic acid anions in the fluids can be used as a source of base [22]. The effectiveness of various organic acids on LA correction is quite different, which is worthy of mention. Normal saline (NS) is often used as an initial resuscitation fluid in clinical settings. There is no doubt that NS is a reasonable alternative to restore perfusion if no other fluid is available. However, massive administration of NS often induces hyperchloremic acidosis [24, 25]. Recent studies suggest that NS may increase acidosis and the incidence of kidney injury in healthy volunteers or critically ill adults, mainly because of renal microvascular contraction induced by hyperchloremia [26, 27]. Therefore, the European guideline on management of major bleeding and coagulopathy following trauma (fourth edition) suggests that excessive use of NS be avoided (Grade $2 \mathrm{C})$, although NS infusion with the potential to restore $\mathrm{pH}$ may be advantageous [6]. Metabolizable anions, such as lactate, acetate and malate, have been included in IV balanced solutions to avoid hyperchloremic acidosis. However, their effects in the treatment of LA vary and have not been compared rigorously. Here, we reviewed the composition of common fluids and their efficiency in LA correction in shock resuscitation.

\section{Lactated Ringer's solution (LR)}

The IV infusion of LR has been regarded as the standard regime and is widely used in the treatment of ICU patients, especially patients with acute massive hemorrhage. Yuan et al. [28] found that LR could alleviate brain trauma-precipitated LA in moderate-HS rats without the increase of arterial lactate levels, although a large amount of lactate was infused. Despite these advantages, some studies indicated LR-induced inflammation and hepatic apoptosis [29] and other studies even questioned its effect on acidosis correction [30]. Recently, it was demonstrated that LR infusion might be detrimental in resuscitation of severe HS in rats [31, 32]. In severe HS, lactate metabolism may be disturbed and the accumulated lactate can be further increased by LR infusion [32,33], which aggravates the inhibition of glycolysis and affects organ function. The damaged liver and kidneys will further inhibit gluconeogenesis and cause lactate accumulation. Therefore, LR mainly affords plasma volume expansion, rather than improves acid-base disturbance. Although there is a lack of clinical evidence available, a large amount of LR infusion may exacerbate lactate accumulation in the resuscitation of severe or decompensated shock in ICU patients. In addition, the plasma lactate level is used as one of the diagnostic parameters in shock severity; thus, LR infusion may interfere with the diagnosis and treatment. Accordingly, the German guideline S3 for Patients with Severe 
and Multiple Injuries states that "the use of LR no longer appears to be worthy of recommendation".

\section{Acetated Ringer's solution (AR)}

Historically, sodium acetate has been used as a fluid bath for hemodialysis [34]. Recently, AR, whose main component is sodium acetate, has been prevalently used in fluid therapy with both crystalloids and colloids in clinical resuscitation. It showed a significantly improved outcome (prolonged survival and less organ injury) in a rat model of severe HS compared with LR [33]. More importantly, AR showed a positive influence on the acid-base disturbance [35] because sodium acetate could alkalize plasma as quickly as SB. Acetate can be metabolized, via thiokinase consumption of 2 ATP molecules, to acetyl-CoA, which is later oxidized in the tricarboxylic acid (TCA) cycle. Acetate can also be converted to bicarbonate in the liver faster than lactate to raise the $\mathrm{pHa}[36,37]$. Furthermore, acetate is mainly degraded in the muscle, which is more ubiquitous than lactate degraded in the liver and kidneys. The metabolic velocity was the same rate as it was administered [38], even though the liver was damaged [39]. In addition, compared with LR, the administration of acetate-buffered solution did not show an elevated lactate concentration in Landrace pigs [40]. Therefore, the superiority of AR to LR is that AR does not inhibit glycolysis. However, impaired cardiac contractile response is a side effect of acetate if a large amount is infused [41]. Further, there was a case report of a surgical patient who first presented lactic acidosis from infusion of a higher load of sodium acetate $(2 \mathrm{~L} / 2 \mathrm{~h})$, which likely delayed lactate clearance and inhibited the activity of PDH, the core enzyme of the TCA cycle [42]. Accordingly, AR is also not promising for correcting LA in critical care patients.

\section{Malate solution}

Malic acid, in the form of its anion malate, is a trigger for the oxidation of acetyl-CoA and could increase the TCA metabolism [43]. Malate is a key anion in Jonosteril Malat (Fresenius Kabi) infusion, which is an appropriate primary fluid therapy for critical care in subjects with moderate and severe acidosis to maintain the perioperative fluid balance [44]. The intragastric administration of malate increased mitochondrial respiration and energy production in rats [45]. Resuscitation with malate also corrected LA in severe HS rats (MAP levels were maintained at $40 \mathrm{mmHg}$ ) [46]. However, malate cannot be metabolized through glycolysis and exhibits no protection of glycolysis under anaerobic metabolism and, thus, no red blood cell (RBC) protection. It may still not be optimal in the treatment of LA under fatal hypoxic conditions.
In all, current fluid therapies can correct LA to some degree, particularly in resuscitation of compensated shock, but the outcome depends on the anion selected in the fluids. The conclusion is that present IV solutions are not promising for LA correction in critically ill patients in ICUs. The pathogenesis of LA should be deeply understood to improve the clinical outcomes. As summarized above, activators of PDH play an important role in correcting LA because of their ability to improve metabolic disorders and decrease lactate accumulation, finally correcting severe acidosis.

\section{Pyruvate as a potential candidate for LA correction}

The present agents for the treatment of LA are non-ideal. A therapeutic approach that can simultaneously correct acidosis and improve organ function with few adverse effects is of clinical importance. Pyruvate, a PDH activator and substrate, is capable of modulating blood acidic $\mathrm{pH}$ by improving metabolic pathways under hypoxia and thus may be an optimal agent in the correction of LA.

\section{Pyruvate and LA correction}

Monocarboxylate pyruvate can effectively correct LA, an effect which has been demonstrated in many preliminary experiments. As early as 1999, Mongan et al. [47] first found that pyruvate resuscitation improved lactate metabolism and prolonged survival in a swine HS model. Later, the phenomena that pyruvate increased $\mathrm{pHa}$ and base excess (BE) and decreased lactate and the lactate/ pyruvate ratio in swine [48] and rats [49] were found one after the other. Sharma et al. [50] showed that resuscitation with hypertonic sodium pyruvate elevated the blood $\mathrm{pH}$ up to nearly 7.42. Petrat et al. [51] studied the effect of pyruvate infusion in a model of rats subjected to severe mesenteric ischemia-reperfusion injury and found that pyruvate raised blood $\mathrm{pH}$ and $\mathrm{BE}$. Koustova et al. [52] and Flaherty et al. [53] presented results that pyruvate Ringer's solution reversed elevated blood lactate levels and preserved organ function in rats subjected to severe HS. Although the concept of pyruvate correction of LA was first proposed in 2004 [54], the conception was not demonstrated until the comparison of pyruvate Ringer's solution with lactate Ringer's solution in fluid resuscitation, in which pyruvate, instead of lactate, efficiently corrected hypoxic LA (increased pHa and $\mathrm{BE}$, decreased blood lactate and the lactate/pyruvate ratio and doubled survival rates) in rats subjected to lethal HS in 2012 [55]. Although clinical data are not available, a case report of Leigh syndrome due to PDH mutation strongly suggests that oral pyruvate even corrects severe LA [56]. The entire reversal of LA by an administration of regular amount of pyruvate within hours in animal models implies its great clinical significance. 
The comparative effects of anions in fluid therapy on LA correction are listed in Table $1[28,31-33,40-42,44-$ $49,51-53,57]$.

\section{Pyruvate superiority in LA correction}

The effect of pyruvate on LA correction is not solely a result of its chemical buffering ability as an alkalizer. Pyruvate has a low dissociation constant $(\mathrm{pKa}=2.49)$, which represents its weaker buffering capacity relative to lactate $(\mathrm{pKa}=3.9)$. Therefore, pyruvate may eliminate LA mainly via its biochemical characteristics in the promotion of energy metabolism and improvement of mitochondrial energetics to oxidize accumulated lactate and consume excess protons.

\section{Improvement the cellular energetics and $\left[\mathrm{H}^{+}\right]$consumption}

Pyruvate is located in the metabolic center of three major substances in mammals, which connect glycolysis in the cytosol and oxidation in the mitochondria in glucose metabolism. It participates in several important metabolic pathways. The major acidosis-eliminating effect of exogenous pyruvate (Sodium Pyruvate, SP) can be illustrated in the following biochemical pathways (Fig. 1).

In the TCA cycle, pyruvate is converted into acetylCoA, catalyzed by the key enzyme PDH complex. Under hypoxic or ischemic conditions, $\mathrm{PDH}$ activity is inhibited. Notably, exogenous pyruvate can effectively restore the inhibited PDH activity through the decline of pyruvate dehydrogenase kinase activity [48] and can enhance the anaplerotic pathway (replenishment of TCA-cycle substrates) by improvement of pyruvate carboxylase (PC). Then, pyruvate is oxidized into $\mathrm{CO}_{2}$ and $\mathrm{H}_{2} \mathrm{O}$ in the TCA cycle, which consumes an equal-molar proton. Therefore, exogenous pyruvate prompts TCA cycle flux and accelerates lactate oxidation and $\left[\mathrm{H}^{+}\right]$consumption, raising the $\mathrm{pHa}$ and producing mitochondrial ATP.

It is reported that shifting the equilibrium of the $\mathrm{LDH}$ pathway towards the production of more lactate results in the consumption of protons and increase of the $\mathrm{NAD}^{+}$/
$\mathrm{NADH}$ (nicotinamide adenine dinucleotide: oxidized form/ reduced form) ratio via the hydrogenation of pyruvate [12]. Pyruvate is endogenously produced during glycolysis by breaking down glucose into two three-carbon molecules. Exogenous pyruvate, as a sole substrate, can be spontaneously converted into lactate by $\mathrm{LDH}$, free from energy [55]. The pyruvate reductive reaction improves the $\mathrm{NAD}^{+} /$ $\mathrm{NADH}$ ratio $[55,58]$, which is essential to sustain the glycolytic pathway and generate glycolytic ATP in anaerobic conditions. The reduction of pyruvate is a systemic alkalinizing reaction with $\left[\mathrm{H}^{+}\right]$consumption, raising the $\mathrm{pHi}$ in hypoxia and even anoxia $[54,59]$. Pyruvate preservation of both the glycolytic pathway and $\mathrm{LDH}$ reduction is a unique superior property relative to other anions, including malate, in IV fluids. This beneficial attribute has recently been further argued by comparison of pyruvate-enriched oral rehydration solution (ORS) with citrate in ORS. Although both pyruvate and citrate in ORS have an equal buffering capacity equivalent to approximately $30 \mu \mathrm{mol}$ of bicarbonate, only pyruvate in ORS can correct LA [60]. Neither bicarbonate nor citrate in ORS corrected hypoxic LA in animals subjected to severe shock.

Pyruvate protects multi-organ function in HS, including that of the liver and kidneys, the main organs for gluconeogenesis metabolism. Thus, exogenous pyruvate as a primary carbon source can enter into and prompt gluconeogenesis, generating glucose and consuming two $\left[\mathrm{H}^{+}\right]$ $[54,61]$. Furthermore, the improved function of heart, liver and kidneys resulting from exogenous pyruvate facilitates tissue oxygenation and lactate clearance.

In addition, pyruvate oxidative metabolism has the lowest oxygen consumption per ATP generation compared to other anions. Lactate oxidation to pyruvate by $\mathrm{LDH}$ requires approximately 0.5 ATP. Acetate oxidation generates $1 / 3$ less ATP, and malate also produces much less ATP in mitochondria compared with equal-molar pyruvate.

Taken together, exogenous pyruvate correction of LA is primarily attributable to its key role in the improvement of glucose metabolism and cellular energetics in

Table 1 The characteristics of different components in fluid therapy

\begin{tabular}{|c|c|c|c|}
\hline \multirow{2}{*}{$\begin{array}{l}\text { Component } \\
\text { in solution }\end{array}$} & \multirow{2}{*}{$\begin{array}{l}\text { Involved metabolic } \\
\text { pathway }\end{array}$} & \multicolumn{2}{|l|}{ Effect on correcting LA } \\
\hline & & Advantage & Deficiency \\
\hline Lactate & $\begin{array}{l}\text { The end-product in the } \\
\text { glycolysis pathway }\end{array}$ & Alleviating LA in moderate HS [28] through plasma expansion & $\begin{array}{l}\text { Not working in severe HS and aggravating } \\
\text { glycolysis inhibition and lactate } \\
\text { accumulation [31-33] }\end{array}$ \\
\hline Acetate & $\begin{array}{l}\text { Metabolized to acetyl-CoA } \\
\text { which is later used in the } \\
\text { TCA cycle }\end{array}$ & $\begin{array}{l}\text { Alkalizing plasma quickly, not inhibiting glycolysis and not } \\
\text { elevating lactate concentration [40] }\end{array}$ & $\begin{array}{l}\text { Side effect of impaired cardiac contractile } \\
\text { response [41], inhibiting PDH activity [42] }\end{array}$ \\
\hline Malate & $\begin{array}{l}\text { The key intermediate in the } \\
\text { TCA cycle }\end{array}$ & $\begin{array}{l}\text { Working in cases of moderate acidosis [44] and moderate } \\
\text { HS [46] and elevating energy production [45] }\end{array}$ & $\begin{array}{l}\text { May not be suitable for the LA under } \\
\text { severe hypoxic conditions }\end{array}$ \\
\hline Pyruvate & $\begin{array}{l}\text { The key intermediate in the } \\
\text { glycolysis pathway and the } \\
\text { TCA cycle }\end{array}$ & $\begin{array}{l}\text { Correcting LA in moderate and lethal HS animals [47-49, 52, } 53 \\
\text { ], isolated failing human myocardium [57] and ischemia-r } \\
\text { eperfusion injury model [51] }\end{array}$ & Not approved in clinic \\
\hline
\end{tabular}




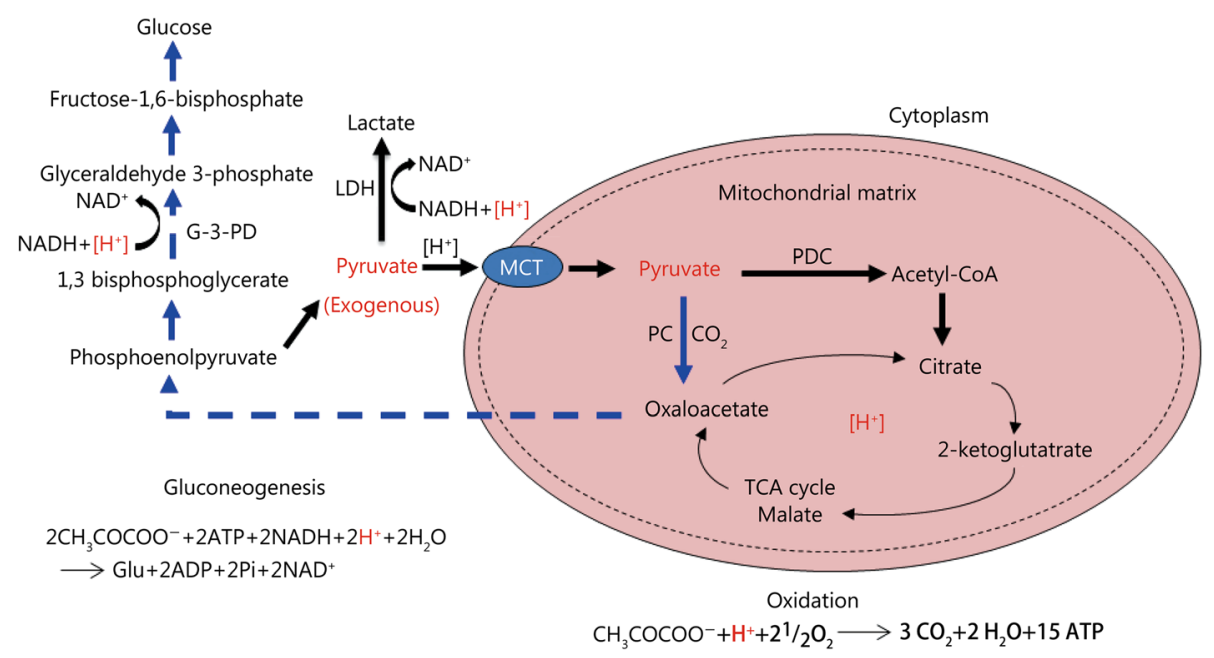

Fig. 1 The metabolic pathways of exogenous pyruvate and their relationships to the consumption of $\left[\mathrm{H}^{+}\right]$First, pyruvate is oxidized into $\mathrm{CO}_{2}$ and $\mathrm{H}_{2} \mathrm{O}$, which consumes an equal-molar $\left[\mathrm{H}^{+}\right]$. Second, when pyruvate is reduced to lactate, the process consumes an equal-molar $\left[\mathrm{H}^{+}\right]$. Finally, the transformation of pyruvate into glucose by gluconeogenesis consumes double-molar $\left[\mathrm{H}^{+}\right] .\left[\mathrm{H}^{+}\right]$: The cytosolic proton enters the mitochondria; $[\mathrm{H}$ +] (red): The proton being consumed in different glucose metabolic pathways; MCT. Monocarboxylate transporters; PDC. Pyruvate dehydrogenase complex; acetyl-CoA. Acetyl coenzyme A; PC. Pyruvate carboxylase; NAD+. Oxidized nicotinamide adenine dinucleotide; NADH. Reduced nicotinamide adenine dinucleotide; LDH. Lactic dehydrogenase; TCA. Tricarboxylic-acid.

hypoxia. The underlying causes that facilitate pyruvate correction of LA may be closely associated with following factors.

Activation of HIF-1a Several studies have demonstrated that pyruvate stabilizes hypoxia-inducible factor$1(\mathrm{HIF}-1 \alpha)$ in normoxic conditions [62, 63]. HIF- $1 \alpha$ is a key transcription factor that up-regulates a series of downstream genes involved in glucose metabolism, promoting the expression of glucose metabolism-related transporters and enzymes, such as glucose transporters [64], hexokinase [65], LDH-A [66] and phosphoglycerate kinase 1 [67]. The up-regulation of these proteins can increase the rates of aerobic glycolysis and enhance lactate oxidation and $\left[\mathrm{H}^{+}\right]$consumption. Moreover, erythropoietin (EPO), a typical up-regulative gene induced by HIF- $1 \alpha$, has cytoprotective and anti-inflammatory functions under various pathological conditions $[68,69]$. It was demonstrated that pyruvate-enriched ORS (Pyr-ORS) significantly preserved the intestinal function and markedly elevated intestinal $E P O$ in the enteral resuscitation of rats with burn injury compared with the effects of citrate-enriched ORS (CitORS) [70]. Therefore, pyruvate may promote the correction of LA via its activation of the HIF- $1 \alpha$-EPO signaling pathway and improvement of cell metabolism.

Anti-oxidation / inflammation Increased reactive oxygen species (ROS) lead to a cascade of events, including protein and fatty acid oxidation, and then suppress PDH activity during hemorrhage, trauma and sepsis. Pyruvate is a natural scavenger of oxidative/nitrosative stress. It directly reacts with free oxygen/nitrogen radicals, which generate in hypoxia, in a non-enzymatical and stochiometrical manner. It also exerts its antioxidant effects by increasing redox potentials in an indirect way [71, 72]. The anti-oxidative and anti-inflammatory effects may protect multi-organ function and, particularly, restore the inhibited PDH activity in hypoxia [49, 73], facilitating LA correction. Pyruvate is superior to the rest of the PDH activators mentioned above because it not only increases PDH activity but also provides PDH substrates in addition to its activation of HIF- $1 \alpha$ and glycolytic protection.

Protection of red blood cell The dual function of RBC is to deliver oxygen to tissues by 2, 3-diphosphoglycerate and to dilate the microvascular system as an oxygen sensor in hypoxic tissues by the release of glycolytic ATP. Moreover, RBC have a strong buffering capacity to sustain the acid-base balance via their $\mathrm{O}_{2}$ - and $\mathrm{CO}_{2}$-transport ability. Pyruvate, in contrast with current anions in IV fluids, protects RBC function by preserving anaerobic glycolytic pathways. For example, preserving fluid containing SP could restore the oxygen-carrying capacity of stored RBC [74]. Pyruvate also protected RBC by sustaining ATP levels and inhibiting activated eNOS activity and NO production during an in vitro cardiopulmonary bypass procedure [75]. Therefore, pyruvate correction of LA may be additionally associated with the improvement of tissue hypoxia through $\mathrm{RBC}$ preservation. The above inference remains to be proven in shock resuscitation with pyruvate. 


\section{Variety of clinical routes of pyruvate administration}

Pyruvate as a component in crystalloid solutions has been often reported [47-53,57]. As a carrier solution in hydroxyethyl starch (HES) 130/0.4, it also showed a beneficial protection of the kidneys in animal resuscitation (J Surg Res. 2018; in press). Recently, various administration routes were studied. Yang et al. [59] reported that IV treatment with a small dose of sodium pyruvate ameliorated metabolic acidosis. Liu et al. [60] reported that Pyr-ORS fully reversed LA and markedly increased survival compared with the effects of Cit-ORS (WHO-ORS II) in dogs with severe burns. In addition, a very low dosage $(<0.2 \mathrm{~g} /$ $\mathrm{kg}$ ) of Pyr-ORS was demonstrated to efficiently correct severe LA in rats with lethal $\mathrm{HS}$ in $4 \mathrm{~h}$, doubling the survival rate [76]. A more recent investigation evidenced again that reduced osmolarity Pyr-ORS, instead of low-osmolar CitORS (WHO-ORS III), effectively corrected LA and significantly elevated survival in rats subjected to lethal burn shock (J Surg Res. 2018; 225: 166-74.). In addition, pyruvate-enriched peritoneal dialysis solution, which was infused into the peritoneal cavity for adjuvant peritoneal resuscitation, was also shown to quickly correct severe acidosis in rats with HS [77].

On the other hand, in the past decade, numerous animal studies have demonstrated that ethyl pyruvate (EP), a derivative of pyruvate, protected multi-organ function with its preferable properties of anti-inflammation and anti-oxidative stress and inhibited elevated lactate in plasma and liver tissue in a septic model [78]. However, there is a distinct difference between EP and SP. The former is an ester, not a salt like the latter, from the chemical point of view. EP is neither a salt nor an alkalizer. EP has to be hydrolyzed via esterase or spontaneous hydrolysis to become pyruvate, resulting in $\left[\mathrm{H}^{+}\right]$ accumulation. Therefore, EP, per se, could not correct metabolic acidosis, even though it reduced hyperlactatemia in rats subjected to septic shock [79].

\section{Conclusions}

To sum up, efficient treatments for severe metabolic acidosis, particularly LA, are of clinical significance because of the severe adverse impacts of acidosis, including impact to cardiovascular function and cellular metabolism and systemic inflammatory reactions, producing a high mortality. Pyruvate is a key energy metabolic substrate and a PDH activator with several unique beneficial biological properties, including anti-oxidative and anti-inflammatory effects and the ability to activate the HIF-1 $\alpha$-EPO signal pathway. Exogenous pyruvate in sodium salt preserves glucose metabolism and cellular energetics superior to the effects of the anions bicarbonate, lactate, acetate and malate in IV fluids in the efficient correction of hypoxic LA by a regular dosage in lethal rodent models. Pyruvate-enriched IV solutions (both crystalloids and colloids) may be not only an agent of volume expansion but also a therapeutic agent for organ dysfunction and metabolic disturbance in clinical fluid resuscitation. Despite of the lack of clinical trials of LA correction by SP at present, a case report strongly indicated its high possibility [56]. Additionally, pyruvate has been applied in preliminary clinical trials through systemic injection of large doses and was shown to be effective and have few side effects [80, 81]. Pyruvate may be safe as a novel therapeutic strategy to correct hypoxic LA accompanied with multi-organ protection in critical care patients. Moreover, a severe concern regarding its instability in solutions has been preliminarily overcome with patents of stable pyruvate aqueous solutions in both China and the USA. Although data from animal and clinical trials with stable pyruvate solutions by the patented approaches are not available, an IV pyruvate injection from a powder preparation obtained prior to clinical use is currently available to correct LA in clinical trials, as demonstrated in animals in terms of its effectiveness and in humans in respect to its safety. It is likely that pyruvate-based IV solutions will become the third generation following NS and lactate/acetate Ringer's solution in fluid therapy [55]. In addition, Pyr-ORS may be beneficial in peri-operative fluid management and pre-hospital rescue in a large scale, such as in earthquakes and terror attacks. Further studies on pyruvate correction of LA are urgently warranted.

\section{Abbreviations \\ AR: Acetated Ringer's solution; ATP: Adenosine triphosphate; BE: Base excess; Cit-ORS: Citrate-enriched ORS; DAG: Desacylghrelin; DCA: Dichloroacetate; \\ EP: Ethyl pyruvate; EPO: Erythropoietin; HS: Hemorrhagic shock; [H \\ ]: Hydrogen ion; HIF-1a: Hypoxia-inducible factor-1a; ICU: Intensive care unit; IV: Intravenous; LA: Type A lactic acidosis; LDH: Lactate dehydrogenase; \\ LR: Lactated Ringer's solution; MCT: Monocarboxylic acid transporter; $\mathrm{NAD}^{+}$/ NADH: Nicotinamide adenine dinucleotide: oxidized form/reduced form; NS: Normal saline; ORS: Oral rehydration solution; PDH: Pyruvate dehydrogenase; pHi: Intracellular pH; pHa: Arterial pH; Pyr-ORS: Pyruvate- enriched ORS; RBC: Red blood cell; SB: Sodium bicarbonate; SP: Sodium pyruvate; TCA cycle: Tricarboxylic acid cycle; THAM: Tromethamine.}

\section{Funding}

This work was supported by the National Natural Science Foundation of China (No. 81700181, No. 81600148).

\section{Authors' contributions \\ YW conceived the study, collected the data and drafted the manuscript. YH and JY participated in the literature research and the writing of the manuscript. FQZ designed the study and reviewed the manuscript. LZ and $\mathrm{HZ}$ conceived the study and finalized the manuscript. All authors read and approved the final manuscript.}

Ethics approval and consent to participate

Not applicable.

\section{Competing interests}

There are no conflicts of interest were declared. The opinions or assertions contained herein are not a reflection of the view of Fresenius Medical Care, Dialysis Centers at Chicago, IL.

\section{Author details}

${ }^{1}$ Institute of Transfusion Medicine, Academy of Military Medical Sciences, Beijing 100850, China. ${ }^{2}$ Department of Transfusion, Hainan Branch of PLA 
General Hospital, Sanya 572013, Hainan, China. ${ }^{3}$ Fresenius Dialysis Centers at Chicago, Rolling Meadows Facility, Chicago, IL 60008, USA. ${ }^{4}$ Shanghai Sandai Pharmaceutical R\&D Co, Shanghai 201203, China.

Received: 23 November 2017 Accepted: 5 April 2018

Published online: 26 April 2018

\section{References}

1. Sedigh-Ardekani M, Sahmeddini MA, Sattarahmady N, Mirkhani H. Lactic acidosis treatment by nanomole level of spermidine in an animal model. Regul Toxicol Pharmacol. 2014:70(2):514-8.

2. Suetrong B, Walley KR. Lactic acidosis in sepsis: it's not all anaerobic. Chest 2016;149(1):252-61.

3. Abramson D, Scalea TM, Hitchcock R, Trooskin SZ, Henry SM, Greenspan J. Lactate clearance and survival following injury. J Trauma. 1993:35(4):584-9.

4. Manikis P, Jankowski S, Zhang H, Kahn RJ, Vincent JL. Correlation of serial blood lactate levels to organ failure and mortality after trauma. Am J Emerg Med. 1995:13(6):619-22.

5. Nichol AD, Egi M, Pettila V, Bellomo R, French C, Hart G, et al. Relative hyperlactatemia and hospital mortality in critically ill patients: a retrospective multi-Centre study. Crit Care. 2010;14:R25

6. Rossaint R, Bouillon B, Cerny V, Coats TJ, Duranteau J, Fernández-Mondéjar $E$, et al. The European guideline on management of major bleeding and coagulopathy following trauma: fourth edition. Crit Care. 2016;20:100.

7. Nguyen HB, Rivers EP, Knoblich BP, Jacobsen G, Muzzin A, Ressler JA, et al. Early lactate clearance is associated with improved outcome in severe sepsis and septic shock. Crit Care Med. 2004;32:1637-42.

8. Bakker J, Nijsten MW, Jansen TC. Clinical use of lactate monitoring in critically ill patients. Ann Intensive Care. 2013;3(1):12.

9. Levy B. Lactate and shock state: the metabolic view. Curr Opin Crit Care. 2006;12(4):315-21.

10. Kraut JA, Kurtz I. Treatment of acute non-anion gap metabolic acidosis. Clin Kidney J. 2015;8(1):93-9.

11. Cooper DJ, Walley KR, Wiggs BR, Russell JA. Bicarbonate does not improve hemodynamics in critically ill patients who have lactic acidosis. A prospective, controlled clinical study. Ann Intern Med. 1990;112(7):492-8.

12. Acharya AP, Rafi M, Woods EC, Gardner AB, Murthy N. Metabolic engineering of lactate dehydrogenase rescues mice from acidosis. Sci Rep. 2014:4:5189.

13. Nahas GG, Sutin KM, Fermon C, Streat S, Wiklund L, Wahlander S, et al. Guidelines for the treatment of acidaemia with THAM. Drugs. 1998:55(2): 191-224.

14. Stacpoole PW, Wright EC, Baumgartner TG, Bersin RM, Buchalter S, Curry SH, et al. A controlled clinical trial of dichloroacetate for treatment of lactic acidosis in adults. The Dichloroacetate-Lactic Acidosis Study Group. N Engl J Med. 1992:327(22):1564-9.

15. Kraut JA, Madias NE. Treatment of acute metabolic acidosis: a pathophysiologic approach. Nat Rev Nephrol. 2012;8(10):589-601.

16. Jaimes R 3rd, Kuzmiak-Glancy S, Brooks DM, Swift LM, Posnack NG, Kay MW. Functional response of the isolated, perfused normoxic heart to pyruvate dehydrogenase activation by dichloroacetate and pyruvate. Pflugers Arch. 2016:468(1):131-42

17. Kaufmann P, Engelstad K, Wei Y, Jhung S, Sano MC, Shungu DC, et al. Dichloroacetate causes toxic neuropathy in MELAS: a randomized, controlled clinical trial. Neurology. 2006;66(3):324-30

18. James MO, Jahn SC, Zhong G, Smeltz MG, Hu Z, Stacpoole PW. Therapeutic applications of dichloroacetate and the role of glutathione transferase zeta1. Pharmacol Ther. 2017;170:166-80.

19. Ferriero R, Boutron A, Brivet M, Kerr D, Morava E, Rodenburg RJ, et al. Phenylbutyrate increases pyruvate dehydrogenase complex activity in cells harboring a variety of defects. Ann Clin Transl Neurol. 2014;1(7):462-70.

20. Sheriff S, Kadeer N, Friend LA, James JH, Alexander JW, Balasubramaniam A. Des-acyl-ghrelin (DAG) normalizes hyperlactacidemia and improves survival in a lethal rat model of burn trauma. Peptides. 2014;60:1-7.

21. Ferriero R, Manco G, Lamantea E, Nusco E, Ferrante MI, Sordino P, et al. Phenylbutyrate therapy for pyruvate dehydrogenase complex deficiency and lactic acidosis. Sci Transl Med. 2013:5(175):175ra31.

22. Kraut JA, Madias NE. Lactic acidosis: current treatments and future directions. Am J Kidney Dis. 2016;68(3):473-82.

23. Reddy S, Weinberg L, Young P. Crystalloid fluid therapy. Crit Care. 2016;20:59.
24. Hadimioglu N, Saadawy I, Saglam T, Ertug Z, Dinckan A. The effect of different crystalloid solutions on acid-base balance and early kidney function after kidney transplantation. Anesth Analg. 2008;107(1):264-9.

25. Scheingraber S, Rehm M, Sehmisch C, Finsterer U. Rapid saline infusion produces hyperchloremic acidosis in patients undergoing gynecologic surgery. Anesthesiology. 1999:90(5):1265-70.

26. Chowdhury AH, Cox EF, Francis ST, Lobo DN. A randomized, controlled, double-blind crossover study on the effects of $2-\mathrm{L}$ infusions of $0.9 \%$ saline and plasma-lyte 148 on renal blood flow velocity and renal cortical tissue perfusion in healthy volunteers. Ann Surg. 2012;256(1):18-24.

27. Yunos NM, Bellomo R, Hegarty C, Story D, Ho L, Bailey M. Association between a chloride-liberal vs chloride-restrictive intravenous fluid administration strategy and kidney injury in critically ill adults. JAMA. 2012 308(15):1566-72

28. Yuan XQ, Wade CE. Lactated Ringer's solution alleviates brain trauma- precipitated lactic acidosis in hemorrhagic shock. J Neurotrauma. 1993;10(3):307-13.

29. Jaskille A, Koustova $E$, Rhee $P$, Britten-Webb J, Chen $H$, Valeri $C R$, et al. Hepatic apoptosis after hemorrhagic shock in rats can be reduced through modifications of conventional Ringer's solution. J Am Coll Surg. 2006;202(1):25-35

30. Knotzer $H$, Pajk W, Maier S, Dünser MW, Ulmer H, Schwarz B, et al. Comparison of lactated Ringer's, gelatine and blood resuscitation on intestinal oxygen supply and mucosal tissue oxygen tension in haemorrhagic shock. Br J Anaesth. 2006;97(4):509-16.

31. Hussmann B, Lendemans S, de Groot H, Rohrig R. Volume replacement with ringer-lactate is detrimental in severe hemorrhagic shock but protective in moderate hemorrhagic shock: studies in a rat model. Crit Care. 2014;18(1):R5.

32. Rohrig R, Rönn $T$, Lendemans $S$, Feldkamp $T$, de Groot H, Petrat F. Adverse effects of resuscitation with lactated ringer compared with ringer solution after severe hemorrhagic shock in rats. Shock. 2012;38(2):137-45.

33. Rohrig R, Wegewitz C, Lendemans S, Petrat F, de Groot $H$. Superiority of acetate compared with lactate in a rodent model of severe hemorrhagic shock. J Surg Res. 2014;186(1):338-45.

34. McCague A, Dermendjieva M, Hutchinson R, Wong DT, Dao N. Sodium acetate infusion in critically ill trauma patients for hyperchloremic acidosis. Scand J Trauma Resusc Emerg Med. 2011;19:24.

35. Ekblad H, Kero P, Takala J. Slow sodium acetate infusion in the correction of metabolic acidosis in premature infants. Am J Dis Child. 1985;139(7):708-10.

36. Almac E, Aksu U, Bezemer R, Jong W, Kandil A, Yuruk K, et al. The acute effects of acetate-balanced colloid and crystalloid resuscitation on renal oxygenation in a rat model of hemorrhagic shock. Resuscitation. 2012;83(9): 1166-72.

37. Keibl C, Sipos W, Ponschab M, Schlimp CJ. Blood biochemical changes in pigs after infusion with acetate-buffered or lactatebuffered crystalloid solutions. Lab Anim (NY). 2015:44(7):268-73.

38. Kveim M, Nesbakken R, Bredesen JE. Plasma acetate concentrations during canine haemorrhagic shock. Scand J Clin Lab Invest. 1979:39(7):645-52.

39. Nakatani T, Sakamoto $Y$, Ando H, Kobayashi K. Effects of Ringer's acetate solution during transient hepatic inflow occlusion in rabbits. Transplantation. 1995;59(7):952-7.

40. Keibl C, Sipos W, Ponschab M, Schlimp CJ. Blood biochemical changes in pigs after infusion with acetate-buffered or lactate-buffered crystalloid solutions. Lab Anim (NY). 2015:44(7):268-73.

41. Aizawa Y, Ohmori T, Imai K, Nara Y, Matsuoka M, Hirasawa Y. Depressant action of acetate upon the human cardiovascular system. ClinNephrol. 1977:8(5):477-80.

42. McCague AM, Bowman N, Wong DT. Lactic acidosis after resuscitation with sodium acetate. J Surg Res. 2012;173(2):362-4.

43. Wu JL, Wu QP, Yang XF, Wei MK, Zhang JM, Huang Q, et al. L-malate reverses oxidative stress and antioxidative defenses in liver and heart of aged rats. Physiol Res. 2008;57(2):261-8.

44. Waack IN, Himmen S, Mueller F, Rohrig R, Roehrborn F, Teloh JK, et al. LMalate's plasma and excretion profile in the treatment of moderate and severe hemorrhagic shock in rats. Biomed Res Int. 2016;2016:5237148.

45. Wu JL, Wu QP, Peng YP, Zhang JM. Effects of L-malate on mitochondrial oxidoreductases in liver of aged rats. Physiol Res. 2011;60(2):329-36.

46. Dai ZL, Wu J, Meng C, Zeng F, Yang Y, Yao SL. Ringer's malate solution protects against the multiple organ injury and dysfunction caused by hemorrhagic shock in rats. Shock. 2012;38(3):268-74.

47. Mongan PD, Fontana JL, Chen R, Bünger R. Intravenous pyruvate prolongs survival during hemorrhagic shock in swine. Am J Phys. 1999;277(6 Pt 2): H2253-63. 
48. Mongan PD, Karaian J, Van Der Schuur BM, Via DK, Sharma P. Pyruvate prevents poly-ADP ribose polymerase (PARP) activation, oxidative damage, and pyruvate dehydrogenase deactivation during hemorrhagic shock in swine. J Surg Res. 2003;112(2):180-8.

49. Sharma P, Walsh KT, Kerr-Knott KA, Karaian JE, Mongan PD. Pyruvate modulates hepatic mitochondrial functions and reduces apoptosis indicators during hemorrhagic shock in rats. Anesthesiology. 2005;103(1):65-73.

50. Sharma P, Mongan PD. Hypertonic soduim pyruvate solutions is more effective than ringer's ethyl pyruvate in the treatment of hemorrhagic shock. Shock. 2010;33(5):532-40.

51. Petrat $F$, Rönn T, de Groot H. Protection by pyruvate infusion in a rat model of severe intestinal ischemia-reperfusion injury. J Surg Res. 2011;167(2):e93-101.

52. Koustova $E$, Rhee $P$, Hancock $T$, Chen $H$, Inocencio R, Jaskille $A$, et al. Ketone and pyruvate Ringer's solutions decrease pulmonary apoptosis in a rat model of severe hemorrhagic shock and resuscitation. Surgery. 2003;134(2):267-74.

53. Flaherty DC, Hoxha B, Sun J, Gurji H, Simecka JW, Mallet RT, OlivenciaYurvati AH. Pyruvate-fortified fluid resuscitation improves hemodynamic stability while suppressing systemic inflammation and myocardial oxidative stress after hemorrhagic shock. Mil Med. 2010;175(3):166-72.

54. Zhou FQ. Pyruvate in the correction of intracellular acidosis: a metabolic basis as a novel superior buffer. Am J Nephrol. 2005;25(1):55-63.

55. Hu S, Bai XD, Liu XQ, Wang HB, Zhong YX, Fang T, et al. Pyruvate Ringer's solution corrects lactic acidosis and prolongs survival during hemorrhagic shock in rats. J Emerg Med. 2013;45(6):885-93.

56. Koga Y, Povalko N, Katayama K, Kakimoto N, Matsuishi T, Naito E, et al. Beneficial effect of pyruvate therapy on Leigh syndrome due to a novel mutation in PDH E1a gene. Brain and Development. 2012;34(2):87-91.

57. Hasenfuss G, Maier LS, Hermann HP, Lüers C, Hünlich M, Zeitz O, et al. Influence of pyruvate on contractile performance and $\mathrm{Ca}^{2+}$ cycling in isolated failing human myocardium. Circulation. 2002;105(2):194-9.

58. Hegde KR, Kovtun S, Varma SD. Inhibition of glycolysis in the retina by oxidative stress: prevention by pyruvate. Mol Cell Biochem. 2010;343(1-2):101-5.

59. Yang J, Zhao JX, Wang Y, Chen G, Cheng WN, Luo X, et al. Effects of sodium pyruvate on ameliorating metabolic acidosis. Artif Cells Nanomed Biotechnol. 2016:44(1):48-55.

60. Liu R, Hu XH, Wang SM, Guo SJ, Li ZY, Bai XD, et al. Pyruvate in oral rehydration salt improves hemodynamics, vasopermeability and survival after burns in dogs. Burns. 2016:42:797-806.

61. Burns SP, Murphy HC, lles RA, Cohen RD. Lactate supply as a determinant of the distribution of intracellular $\mathrm{pH}$ within the hepatic lobule. Biochem $\mathrm{J}$. 2001;358(Pt3):569-71.

62. Lu H, Forbes RA, Verma A. Hypoxia inducible factor 1 activation by aerobic glycolysis implicates the Warburg effect in carcinogenesis. J Biol Chem. 2002;277(26):23111-5.

63. Dalgard CL, Lu H, Mohyeldin A, Verma A. Endogenous 2-oxoacids differentally regulate expression of oxygen sensors. Biochem J. 2004; 380(Pt2):419-24

64. Maxwell PH, Dachs GU, Gleadle JM, Nicholls LG, Harris AL, Stratford IJ, et al. Hypoxia-inducible factor-1 modulates gene expression in solid tumors and influences both angiogenesis and tumor growth. Proc Natl Acad Sci U S A. 1997;94(15):8104-9.

65. Mathupala SP, Rempel A, Pedersen PL. Glucose catabolism in cancer cells: identification and characterization of a marked activation response of the type II hexokinase gene to hypoxic conditions. J Biol Chem. 2001;276(46):43407-12.

66. Semenza GL, Jiang BH, Leung SW, Passantino $R$, Concordet JP, Maire $P$, et al. Hypoxia response elements in the aldolaseA, enolase 1, and lactate dehydrogenase a gene promoters contain essential binding sites for hypoxia-inducible factor 1. J Biol Chem. 1996;271(51):32529-37.

67. Semenza GL, Roth PH, Fang HM, Wang GL. Transcriptional regulation of genes encoding glycolytic enzymes by hypoxia-inducible factor 1. J Biol Chem. 1994:269(38):23757-63.

68. Yazihan N, Ataoglu H, Kavas GO, Akyurek N, Yener B, Aydın C. The effect of K-ATP channel blockage during erythropoietin treatment in renal ischemiareperfusion injury. J Investig Surg. 2008;21(6):340-7.

69. Baker JE. Erythropoietin mimics ischemic preconditioning. Vasc Pharmacol. 2005;42(5-6):233-41.

70. Hu S, Lin ZL, Zhao ZK, Liu R, Ma L, Luo HM, et al. Pyruvate is superior to citrate in oral rehydration solution in the protection of intestine via hypoxia-inducible factor1 activation in rats with burn injury. J Parenter Enter Nutr. 2016;40(7):924-33.
71. Bassenge E, Sommer O, Schwemmer M, Bünger R. Antioxidant pyruvate inhibits cardiac formation of reactive oxygen species through changes in redox state. Am J Physiol Heart Circ Physiol. 2000;279(5):H2431-8.

72. Tejero-Taldo MI, Caffrey JL, Sun J, Mallet RT. Antioxidant properties of pyruvate mediate its potentiation of $\beta$-adrenergic inotropism in stunned myocardium. J Mol Cell Cardiol. 1999;31(10):1863-72.

73. Saiki Y, Lopaschuk GD, Dodge K, Yamaya K, Morgan C, Rebeyka IM. Pyruvate augments mechanical function via activation of the pyruvate dehydrogenase complex in reperfused ischemic dehydrogenase complex immature rabbit hearts. J Surg Res. 1998;79(2):164-9.

74. Xia S, Chen G, Wang B, Yin Y, Sun Z, Zhao J, et al. Addition of sodium pyruvate to stored red blood cells attenuates liver injury in a murine transfusion model. Mediat Inflamm. 2016;2016:3549207.

75. Gou D, Tan H, Cai H, Zhou F. Pyruvate effects on red blood cells during in vitro cardiopulmonary bypass with dogs' blood. Artif Organs. 2012;36(11): 988-91.

76. Yu W, Hu S, Xie ZY, He ZJ, Luo HM, Lin HY, et al. Pyruvate oral rehydration solution improved visceral function and survival in shock rats. J Surg Res. 2015;193(1):344-54.

77. Lu XG, Kang X, Zhou FQ, Wang XZ, Guo S, Fan ZW, et al. Effects of pyruvate-enriched peritoneal dialysis solution on intestinal barrier in peritonealresuscitation from hemorrhagic shock in rats. J Surg Res. 2015; 193(1):368-76.

78. Kang H, Mao Z, Zhao Y, Yin T, Song Q, Pan L, et al. Ethyl pyruvate protects against sepsis by regulating energy metabolism. Ther Clin Risk Manag. 2016; 23(12):287-94.

79. Venkataraman R, Kellum JA, Song M, Fink MP. Resuscitation with Ringer's ethyl pyruvate solution prolongs survival and modulates plasma cytokine and nitrite/nitrate concentrations in a rat model of lipopolysaccharideinduced shock. Shock. 2002;18(6):507-12.

80. Mateva L, Petkova I, Petrov K, Beniozef D, Bojilova M, Vankova L, et al. Tenday course of sodium pyruvate infusions in patients with chronic liver diseases (CLD). Jpn Pharmacol Ther. 1996;24(12):2629-39.

81. Schillinger W, Hünlich M, Sossalla S, Hermann HP, Hasenfuss G. Intracoronary pyruvate in cardiogenic shock as an adjunctive therapy to catecholamines and intra-aortic balloon pump shows beneficial effects on hemodynamics. Clin Res Cardiol. 2011;100(5):433-8.

\section{Ready to submit your research? Choose BMC and benefit from:}

- fast, convenient online submission

- thorough peer review by experienced researchers in your field

- rapid publication on acceptance

- support for research data, including large and complex data types

- gold Open Access which fosters wider collaboration and increased citations

- maximum visibility for your research: over $100 \mathrm{M}$ website views per year

At BMC, research is always in progress.

Learn more biomedcentral.com/submissions 